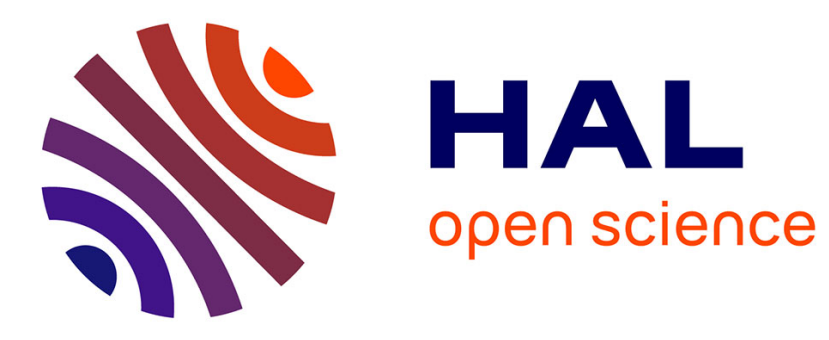

\title{
Stability Criteria for Asynchronous Sampled-data Systems - A Fragmentation Approach
}

Corentin Briat, Alexandre Seuret

\section{To cite this version:}

Corentin Briat, Alexandre Seuret. Stability Criteria for Asynchronous Sampled-data Systems - A Fragmentation Approach. IFAC WC 2011 - 18th IFAC World Congress, Aug 2011, Milan, Italy. pp.6. hal-00575532

\section{HAL Id: hal-00575532 https://hal.science/hal-00575532}

Submitted on 10 Mar 2011

HAL is a multi-disciplinary open access archive for the deposit and dissemination of scientific research documents, whether they are published or not. The documents may come from teaching and research institutions in France or abroad, or from public or private research centers.
L'archive ouverte pluridisciplinaire HAL, est destinée au dépôt et à la diffusion de documents scientifiques de niveau recherche, publiés ou non, émanant des établissements d'enseignement et de recherche français ou étrangers, des laboratoires publics ou privés. 


\title{
Stability Criteria for Asynchronous Sampled-data Systems - A Fragmentation Approach
}

\author{
C. Briat* \\ * ACCESS Linnaeus Centre, Division of Optimization and Systems \\ Theory, KTH, SE-10044, Stockholm, Sweden; e-mail: \\ corentin@briat.info,cbriat@math.kth.se,http://www.briat.info. \\ A. Seuret ${ }^{* *}$ \\ ** GIPSA-lab, NeCS INRIA Project-Team - Control Systems \\ Department - INP BP 46, Domaine Universitaire - 38400 Saint \\ Martin d'Heres - FRANCE.
}

\begin{abstract}
The stability analysis of asynchronous sampled-data systems is studied. The approach is based on a recent result which allows to study, in an equivalent way, the quadratic stability of asynchronous sampled-data systems in a continuous-time framework via the use of peculiar functionals satisfying a necessary boundary condition. The method developed here is an extension of previous results using a fragmentation technique inspired from recent advances in time-delay systems theory. The approach leads to a tractable convex feasibility problem involving a small number of finite dimensional LMIs. The approach is then finally illustrated through several examples.
\end{abstract}

Keywords: asynchronous sampled-data systems; robustness; LMIs

\section{INTRODUCTION}

Sampled-data systems have emerged quite recently when digital controllers began to be used [Chen and Francis, 1995] and consist in continuous-time systems controlled, most of the time, by a piecewise constant control input, generally computed by digital devices like microprocessors coupled with I/O devices. A somehow reasonable assumption, which was to consider a constant sampling period, has led to a well-rounded theory and a wide panel of applications. However, in more recent applications, jitter, computation delays, possible delays on the input/output ${ }^{1}$ and data losses (arising in Networked Control Systems [Zhang et al., 2001, Hespanha et al., 2007]) can affect the regularity of the sampling period and may lead to an unstable behavior for the closed-loop system. To analyze and overcome this problem of stability deterioration, several approaches have therefore been developed.

Several works have been devoted to the stability analysis of such systems. In [Fridman et al., 2004, Suplin et al., 2007], an input-delay based technique is considered where the sampled state is identified to a delayed state as $x\left(t_{k}\right)=x(t-\tau(t))$ where $\tau(t)=t-t_{k}, t \in\left[t_{k}, t_{k+1}\right)$. It is clear that the delay value is bounded from above by $T$, the maximal sampling period. Thus delay-dependent stability

\footnotetext{
$\star$ This work was supported by the ACCESS and RICSNET projects. ${ }^{\star \star}$ This research is supported by FeedNetBack project, FP7- ICT2007-2 and CONNECT project, PSIROB06-174215

1 which are most of the time not commensurable with the sampling period
}

results can be used. However, the actual sawtooth shape of the delay can be difficult to handle in that framework. Continuous-time techniques based on impulsive/hybrid systems [Naghshtabrizi et al., 2008, Seuret, 2009] use functionals depending explicitly on $\tau(t)$. Due to the discontinuity of $\tau(t)$ at sampling times $t_{k}, k \in \mathbb{N}$, the functionals were built in such a way that the discontinuous terms vanish at sampling instants. Such functionals also have the benefits of considering exactly the constraint $\dot{\tau}(t)=1$ between sampling instants. Robust techniques in continuous-time have also been employed [Mirkin, 2007, Fujioka, 2009b] and in discrete-time [Montestruque and Antsaklis, 2004, Oishi and Fujioka, 2009, Fujioka et al., 2010] using smallgain, passivity, IQCs and robust optimization techniques.

The approach considered here is based on recent results on sampled-data systems analyzed using impulsive/hybrid systems theory. It lies in the continuity of the recent works [Seuret, 2010] in which the stability analysis was done in a more general way. Indeed, in this paper, a necessary condition the functionals must satisfy is considered and the engendered class includes the functionals used in [Naghshtabrizi et al., 2008, Seuret, 2009] as particular cases. A very fundamental feature of this result is the emphasis of the equivalency between continuousand discrete-time analysis. Indeed, it turns out that the decrease of the continuous-time functionals is equivalent to the decrease of the discrete-time Lyapunov function at each sampling instants. A consequence of this result consists in the relaxation of some positive definite ma- 
trices $^{2}$ to indefinite ones. This makes the result more flexible via the introduction of new degrees of freedom and reduces the conservatism. Moreover, the necessary conditions also provides a guideline for the construction of new functionals.

According to this result, it turns out that this class of functionals may contain integral quadratic terms leading then to integral terms in the stability conditions. A way to get rid of these integral is the use of the Jensen's inequality, similarly as for time-delay systems. Thus, following the ideas of [Gouaisbaut and Peaucelle, 2006, .Gouaisbaut and Peaucelle, 2006, Briat, 2008] where it has been emphasized that fragmentation of integral terms in such functionals are very often related to an efficiency increase, the same procedure is then applied here to the functional considered in [Seuret, 2010]. This leads to a sequence of results providing sufficient conditions for the asymptotic stability of asynchronous linear sampled-data systems.

The paper is structured as follows. In Section 2, the problem is formulated. Section 3 states a fundamental result on the equivalence between the stability notions in discrete- and continuous-time. This result is then applied to sampled-data systems in Section 4. Finally, some examples illustrate the approach in Section 5.

The notations are quite standard. Given two symmetric matrices $A, B, A \succ B$ stands for $A-B$ positive definite. The identity matrix of dimension $n$ is denoted by $I_{n}$ while $0_{m \times p}$ is the 0 matrix of dimension $m \times p$. A matrix obtained by stacking matrices $U_{0}, U_{1}, \ldots, U_{n}$ with $U_{0}$ on the top is denoted by $\operatorname{col}_{i=0}^{n}\left\{U_{i}\right\}$. For a given function $x: \mathbb{R}_{+} \rightarrow \mathbb{R}^{n}$ and an increasing sequence $\left\{t_{k}\right\}_{k \in \mathbb{N}}$ in $\mathbb{R}_{+}$, the function $\varkappa_{k}$ is defined, for $k \in \mathbb{N}$, as

$$
\begin{aligned}
\varkappa_{k}:\left[0, T_{k}\right] & \rightarrow \mathbb{R}^{n} \\
\tau & \rightarrow x\left(t_{k}+\tau\right) .
\end{aligned}
$$

The set of continuous functions mapping $[0, T]$ to $\mathbb{R}^{n}$ is denoted by $C\left([0, T], \mathbb{R}^{n}\right)$. If $T_{k} \leq T, k \in \mathbb{N}$ and $x \in$ $C\left(\mathbb{R}_{+}, \mathbb{R}^{n}\right)$ then obviously $\varkappa_{k} \in \bar{C}\left([0, T], \mathbb{R}^{n}\right)$. Implicitly defined symmetric elements in symmetric matrices are denoted by $\star$.

\section{PROBLEM FORMULATION}

Consider the LTI continuous-time system

$$
\dot{x}(t)=A x(t)+B u(t)
$$

where $x \in \mathbb{R}^{n}$ and $u \in \mathbb{R}^{m}$ are the system state and the control input respectively. In this paper, we are interested in aperiodic sampled-data based state-feedback control laws of the form

$$
u(t)=K x\left(t_{k}\right), t \in\left[t_{k}, t_{k+1}\right)
$$

where $K$ is the controller gain. The time between two successive sampling instants is defined as $T_{k}:=t_{k+1}-t_{k}$ and satisfies $0<T_{k}<T$. By integration of (1)-(2) over $\left[t_{k}, t_{k}+\tau(t)\right], \tau(t)=t-t_{k}$, we get

$$
\begin{aligned}
& x\left(t_{k}+\tau(t)\right)=\tilde{A}(\tau(t)) x\left(t_{k}\right) \\
& \tilde{A}(\tau(t))=e^{A \tau(t)}+\int_{0}^{\tau(t)} e^{A(\tau(t)-\theta)} d \theta B K
\end{aligned}
$$

\footnotetext{
2 whose positive definiteness was required by previously developed theorems
}

for all $t \in\left[t_{k}, t_{k+1}\right]$ and $\tau(t) \in\left[0, T_{k}\right]$. When the sampling period is constant (i.e. $T_{k}=T$ for all $k \in \mathbb{N}$ ), then the stroboscopic model is given by

$$
x\left(t_{k+1}\right)=\tilde{A}(T) x\left(t_{k}\right) .
$$

It is well-known that the above discrete-time LTI system is asymptotically stable if and only if the eigenvalues of the stroboscopic map $\tilde{A}(T)$ lie within the unit disc. When the sampling period is time-varying, the above condition true for any sampling period is not sufficient anymore. Indeed, it is possible to find distinct Schur matrices $M_{1}, M_{2}$ such that their product $M_{1} M_{2}$ is not. A way to overcome this difficulty is to use discrete-time Lyapunov stability analysis tools; see e.g. [Suh, 2008, Oishi and Fujioka, 2009, Fujioka et al., 2010] where constant or switched discretetime Lyapunov functions are considered.

\section{EQUIVALENCE BETWEEN CONTINUOUS- AND DISCRETE-TIME STABILITY}

In this section, the fundamental question on the equivalence of stability notions in discrete- and continuoustime is considered. Indeed, since sampled-data systems are analyzed in a continuous-time framework while, from the stroboscopic point of view, they are discrete-time systems, it seems important to clarify the correspondence between the two domains. The following theorem states this correspondence through the existence of a functional satisfying a necessary boundary condition.

Theorem 1. [Seuret, 2010] Let $V: \mathbb{R}^{n} \rightarrow \mathbb{R}^{+}$be a function for which there exist real numbers $\mu_{1}, \mu_{2}, 0<\mu_{1}<\mu_{2}<$ $+\infty$ and an integer number $p>0$ such that

$$
\forall \eta \in \mathbb{R}^{n}, \quad \mu_{1}\|\eta\|^{p} \leq V(\eta) \leq \mu_{2}\|\eta\|^{p} .
$$

Then the two following statements are equivalent:

(i) $\forall k \geq 0, \quad \Delta V(k)=V\left(x\left(t_{k+1}\right)\right)-V\left(x\left(t_{k}\right)\right)<0$;

(ii) There exists a continuous functional $V_{1}: \mathbb{R} \times$ $C([0, T], \mathbb{R}) \rightarrow \mathbb{R}$, differentiable over $\left[t_{k} t_{k+1}\right)$ satisfying

$$
V_{1}\left(T_{k}, \varkappa_{k}\right)=V_{1}\left(0, \varkappa_{k}\right)
$$

for all $k \in \mathbb{N}$ and such that the functional

$$
\mathcal{W}\left(\tau(t), \varkappa_{k}\right):=V(x(t))+V_{1}\left(\tau(t), \varkappa_{k}(\tau(t))\right)
$$

satisfies

$$
\dot{\mathcal{W}}\left(\tau(t), \varkappa_{k}\right)=\frac{d}{d t} \mathcal{W}\left(\tau(t), \varkappa_{k}\right)<0
$$

for all $\tau \in\left[0, T_{k}\right], k \in \mathbb{N}-\{0\}$.

Moreover, if one of these two statements is satisfied, the solutions of system (1)-(2) are asymptotically stable.

Proof :

In the following, we drop the dependence on time $t$ for simplicity. Let us consider a given time interval $\left[t_{k}, t_{k+1}\right)$, $k \in \mathbb{N}$.

Proof of (ii) $\Rightarrow$ (i). Assume (ii) is satisfied, a simple integration of inequality (7) over $\left[t_{k}, t_{k+1}\right]$ implies (i) using the boundary condition (6).

Proof of (i) $\Rightarrow$ (ii). Assume now (i) holds. Following the idea of [Peet et al., 2009, Lemma 2], define the functional

$$
V_{1}\left(\tau, \varkappa_{k}\right)=-V(x)+\tau / T_{k} \Delta V(k) .
$$

Note that $V_{1}$ is indeed a functional since it involves $\Delta V(k)$ which depends on $x\left(t_{k}\right)=\varkappa_{k}(0), x\left(t_{k+1}\right)=\varkappa_{k}\left(T_{k}\right)$ 
and $x(t)=\varkappa_{k}\left(t-t_{k}\right)$ for all $t \in\left[t_{k}, t_{k+1}\right]$. Simple computations show that this functional satisfies (6) and after substitution into (7) we get

$$
\dot{\mathcal{W}}=\frac{1}{T_{k}} \Delta V(k)<0 .
$$

Hence we have $\Delta V(k)<0$ and (i) holds. The equivalence is proved.

From the discrete-time Lyapunov theorem, the equilibrium of the discrete-time system is asymptotically stable. Consequently, $x\left(t_{k}\right)$ tends to zero as $t_{k}$ tends to infinity.

The end of the proof consists in ensuring that the Lyapunov function $V(x(t) s)$ remains bounded between sampling instants, i.e. for $t \in\left[t_{k}, t_{k+1}\right)$. From (5), we have $V(x(t)) \leq \mu_{2}\left\|\tilde{A}(\tau) x\left(t_{k}\right)\right\|^{p}$ for any $\tau \in\left[0, T_{k}\right] \subset[0, T]$, $t \in\left[t_{k}, t_{k+1}\right)$. Since the function

$$
\tilde{A}:[0, T] \rightarrow \mathbb{R}^{n \times n}
$$

is continuous and bounded over $[0, T]$ then there exists a positive scalar $\mu_{m}>0$ such that $\|\tilde{A}(\theta)\| \leq \mu_{m}$ for all $\theta \in[0, T]$. This implies that $V(x(t)) \leq \mu_{2} \mu_{m}^{p}\left\|x\left(t_{k}\right)\right\|^{p}$ and that the continuous-time Lyapunov function $V(x(t))$ is bounded between sampling instants. Hence, $V(x(t))$ converges uniformly asymptotically to 0 when $t \rightarrow+\infty$. The proof is complete.

Remark 3.1. When a discrete-time quadratic Lyapunov function $V(x)=x^{T} P x$ with $P=P^{T} \succ 0$ is chosen, then statement (i) is equivalent to saying that

$$
\tilde{A}(\theta)^{T} P \tilde{A}(\theta)-P \prec 0
$$

for all $\theta$ in the interval of admissible (time-varying) sampling periods. Note that the above problem is a semiinfinite dimensional convex feasibility problem which is difficult to solve due to the presence of exponential terms in $\tilde{A}(T)$ [Hetel et al., 2008, Fujioka, 2009a, Oishi and Fujioka, 2009]. As we shall see later, the obtained LMI conditions using statement (ii) are still semi-infinite but linear in the sampling-period and hence easy to check. They provide thus a good alternative to the discretetime stability condition provided that the functional $V_{1}$ is suitably chosen.

Remark 3.2. The reason for introducing functionals is simple. Indeed, since continuous-time methods are employed for discrete-time systems, we need to consider the intersample behavior. The intersample behavior of the state is exactly considered by choosing the functions $\varkappa_{k}$ as the state of the continuous-time comparison system. Hence the comparison system is an infinite dimensional system. Similar ideas have been raised for the analysis of sampleddata systems [Chen and Francis, 1990].

It is important to note that if a continuous-time quadratic Lyapunov function (i.e. $V(x(t))=x(t)^{T} P x(t)>0$ and $\dot{V}(x(t))<0$ for $x(t) \neq 0$ and $\left.t \in \mathbb{R}_{+}\right)$is used to prove the stability of system (1)-(2), the obtained conditions can be extremely conservative. Indeed, it is not difficult to show that, when considering the stability of sampleddata systems in a continuous-time framework, the above continuous-time function may be locally increasing even if the sampled-data system is asymptotically stable. What really matters is the decrease of the Lyapunov function when evaluated at the sampling instants (the standard

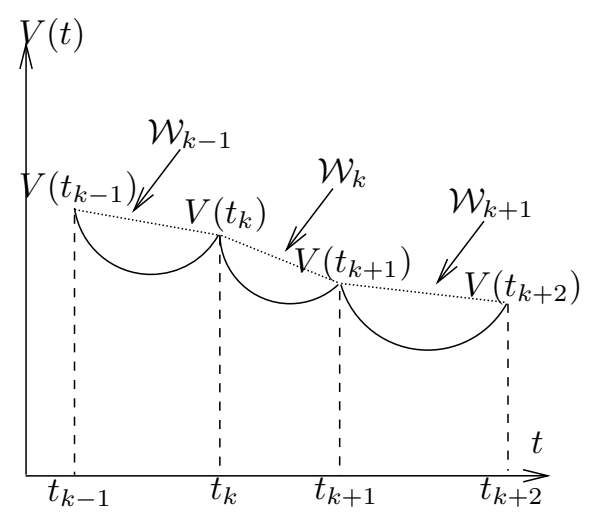

Fig. 1. Illustration of the proof of Theorem 1 where $\mathcal{W}_{k}:=$ $\mathcal{W}\left(t-t_{k}, \varkappa_{k}\right)$

discrete-time Lyapunov condition). The indefinite functional $V_{1}$ relaxes the continuous-time constraint $\dot{V}<0$ into $\dot{V}+\dot{V}_{1}<0$ allowing $V$ to be locally increasing, as illustrated in Fig. 1.

In the literature, several works introduced functionals with different $V_{1}$ satisfying the necessary conditions of Theorem 1 [Naghshtabrizi et al., 2008, Fridman, 2010, Seuret, 2009]. However, these results are based on Lyapunov-Krasovskiilike theorems and the involved $V_{1}$ had to be positive. Theorem 1 says that this positiveness is not necessary as long as $V_{1}$ satisfies the boundary condition (6). Finally, it is also important to point out that no direct relationship between the continuous- and discrete-time results was emphasized in these previous works.

\section{ASYMPTOTIC STABILITY OF ASYNCHRONOUS SAMPLED-DATA SYSTEMS}

Let us consider the asynchronous sampled-data linear system (1)-(2) with sampling period in the interval $\left[T^{-}, T^{+}\right]$ where $0 \leq T^{-}<T^{+}<+\infty$. We will consider the standard quadratic Lyapunov function

$$
V(x)=x^{T} P x
$$

for the discrete-time stability conditions. The goal is then to provide a sufficient condition for $\Delta V(k)=V\left(x\left(t_{k+1}\right)\right)-$ $V\left(x\left(t_{k}\right)\right)<0$ for all $T^{-} \leq t_{k+1}-t_{k} \leq T^{+}$. This problem falls into the framework of Theorem 1 and hence admits an equivalent continuous-time formulation.

In order to refine the stability conditions proposed in [Seuret, 2010], a fragmentation of the interval $\left[t_{k}, t\right]$ in $N>0$ disjoint parts is performed and a functional is built for the obtained fragmented system. Using such a formulation the following theorem, providing a sufficient condition for stability of (1)-(2), is obtained.

Theorem 2. The system (1)-(2) is asymptotically stable for any time-varying sampling period in $\left[T^{-}, T^{+}\right]$if there exist constant matrices $P=P^{T} \succ 0, R_{i}=R_{i}^{T} \succ 0$, $i=0, \ldots, N-1$ and $U=U^{T} \in \mathbb{R}^{n \times n}, S=S^{T} \in \mathbb{R}^{n N \times n N}$, $Q \in \mathbb{R}^{n N \times n}$ and $Y \in \mathbb{R}^{n(N+1) \times n N}$ such that the LMIs

$$
\begin{aligned}
& \Psi_{1}+T^{-}\left(\Psi_{2}+\Psi_{3}\right) \prec 0, \quad \Psi_{1}+T^{+}\left(\Psi_{2}+\Psi_{3}\right) \prec 0 \\
& {\left[\begin{array}{cc}
\Psi_{1}-T^{-} \Psi_{3} & T^{-} Y \\
\star & -\alpha^{-} \bar{R}
\end{array}\right] \prec 0, \quad\left[\begin{array}{cc}
\Psi_{1}-T^{+} \Psi_{3} & T^{+} Y \\
\star & -\alpha^{+} \bar{R}
\end{array}\right] \prec 0}
\end{aligned}
$$

hold where $\alpha^{-}=N T^{-}, \alpha^{+}=N T^{+}$and 


$$
\begin{aligned}
& \Psi_{1}=2 N_{0}^{T} P M_{0}-\Lambda_{12}^{T}\left[S \Lambda_{12}+2 Q N_{2}\right]-2 Y \Lambda_{12} \\
& \left.\Psi_{2}=M^{T} \tilde{R} M+2\left(\Lambda_{12} \Delta_{N}^{2} M\right)^{T} S \Lambda_{12}+2 Q \Lambda_{2}\right] \\
& \Psi_{3}=N_{2} U N_{2}, \quad \bar{R}=\operatorname{diag}\left\{R_{i}\right\}_{i=0, \ldots, N-1} \\
& \tilde{R}=\Delta_{N}^{T}\left[\Lambda_{1}^{T} \bar{R} \Lambda_{1}-\Lambda_{2}^{T} \bar{R} \Lambda_{2}\right] \Delta_{N}
\end{aligned}
$$

with

$$
\begin{aligned}
& M_{i}=\left[\begin{array}{llll}
0_{n \times i n} & A & 0_{n \times(N-i-1) n} & B K
\end{array}\right] \\
& M=\underset{i=0}{\operatorname{col}}\left\{M_{i}\right\}, \quad N_{0}=\left[\begin{array}{ll}
I_{n} & 0_{n \times N n}
\end{array}\right] \\
& N_{2}=\left[\begin{array}{ll}
0_{n \times N n} & I_{n}
\end{array}\right] \\
& \left.\Lambda_{1}=I_{(N+1) n} 0_{(N+1) n \times n}\right] \\
& \Lambda_{2}=\left[\begin{array}{ll}
0_{(N+1) n \times n} & I_{(N+1) n}
\end{array}\right] \Lambda_{12}=\Lambda_{1}-\Lambda_{2} \\
& \Delta_{N}=\underset{i=0, \ldots, N}{\operatorname{diag}}\left\{\sqrt{\frac{N-i}{N}} I_{n}\right\} \text {. }
\end{aligned}
$$

Proof : The proof is divided in 4 parts. The first one concerns the fragmentation of the interval $\left[t_{k}, t\right]$ in $N$ parts and the derivation of the 'fragmented' system. The second one addresses the construction of a suitable functional $V_{1}$ complying with the fragmentation. The third part is essentially tedious calculations, mainly consisting of the differentiation of $V+V_{1}$ and the bounding of integral terms to finally obtained semi-infinite matrix inequalities. At end, the fourth part discusses how to equivalently turn to problem into a finite dimensional convex feasibility problem involving LMIs.

Part 1. Let us consider a sampling instant $t_{k}$ and the partition of the corresponding interval $\left[t_{k}, t\right]$, into $N$ subintervals, by considering the fragmented sampling instant $t_{k}^{i}\left(t, t_{k}\right)$ defined as

$$
t_{k}^{i}(t)=t_{k}+\frac{N-i}{N}\left(t-t_{k}\right)=\frac{i}{N} t_{k}+\frac{N-i}{N} t .
$$

Note that the $t_{k}^{i}$ 's lie in the interval $\left[t_{k}, t\right]$ since they are defined as convex combinations of the bounds on the interval. Define the variable $x^{i}(t), i=1, \ldots, N$ to be

$$
x^{i}\left(t, t_{k}\right):=x\left(t_{k}^{i}(t)\right)=x\left(t_{k}+\frac{N-i}{N}\left(t-t_{k}\right)\right)
$$

for all $t \in\left[t_{k}, t_{k+1}\right)$. It is clear that the $x^{i}$ 's represent a fragmentation of the variable $x$ over the interval $\left[t_{k}, t\right) \subset$ $\left[t_{k}, t_{k+1}\right]$. Their derivative is given by

$$
\frac{d}{d t}\left\{x\left(t_{k}^{i}(t)\right)\right\}=\frac{N-i}{N}\left(A x^{i}\left(t, t_{k}\right)+B K x\left(t_{k}\right)\right)
$$

and we define $\dot{x}\left(t_{k}^{i}(t)\right)$ as

$$
\dot{x}\left(t_{k}^{i}(t)\right)=A x^{i}\left(t, t_{k}\right)+B K x\left(t_{k}\right) .
$$

The augmented vector $X(t)$ is defined as

$$
X(t)=\underset{\substack{i=0, \ldots, N \\ \operatorname{col}}}{ }\left\{x^{i}\left(t, t_{k}\right)\right\}
$$

and the vector $\dot{X}$ as

$$
\begin{aligned}
\dot{X}(t) & \left.=\underset{\substack{i=0, \ldots, N \\
\operatorname{col}}}{x}\left(t_{k}^{i}(t)\right)\right\} \\
& =M X(t)
\end{aligned}
$$

where $M$ is defined in (13). The time-derivative of $X(t)$ obeys

$$
\begin{aligned}
\frac{d}{d t}\{X(t)\} & =\underset{\substack{i=0, \ldots, N \\
\text { col }}}{d}\left\{\frac{d}{d t}\left\{x\left(t_{k}^{i}(t)\right)\right\}\right\} \\
& =\frac{d}{d t}\{X(t)\}=\Delta_{N}^{2} M X(t)
\end{aligned}
$$

where $M$ and $\Delta_{N}$ are defined in (13).

Part 2. From now on, the dependence on time will be dropped when no confusion arises and the shorthand $x_{k}^{i}$ will be used to denote $x^{i}\left(t, t_{k}\right)$. Let us introduce the column vector $\zeta_{k}$ as

$$
\zeta_{k}=\underset{i=0, \ldots, N-1}{\operatorname{col}}\left\{x_{k}^{i}-x_{k}^{i-1}\right\} .
$$

and the functional

$$
\begin{aligned}
V_{1}\left(\tau, \varkappa_{k}\right):= & \left(T_{k}-\tau\right) \zeta_{k}^{T}\left[S \zeta_{k}+2 Q x\left(t_{k}\right)\right] \\
& +\left(T_{k}-\tau\right) \sum_{i=0}^{N-1} \int_{t_{k}^{i-1}(t)}^{t_{k}^{i}(t)} \dot{x}(s)^{T} R_{i} \dot{x}(s) d s \\
& +\left(T_{k}-\tau\right) \tau x\left(t_{k}\right)^{T} U x\left(t_{k}\right)
\end{aligned}
$$

defined for some positive definite matrices $R_{i}=R_{i}^{T} \prec 0$, $i=0, \ldots, N-1$ and symmetric matrices $S=S^{T}, U=U^{T}$. Note that the matrices $U$ and $S$ can be chosen indefinite since this functional satisfies the necessary boundary condition (6), i.e. $V_{1}\left(0, \varkappa_{k}\right)=V_{1}\left(T_{k}, \varkappa_{k}\right)=0$.

Part 3. Following the condition (7), the differentiation of $\mathcal{W}=V+V_{1}$ leads to:

$$
\begin{aligned}
\dot{\mathcal{W}}\left(\tau, \varkappa_{k}\right)= & 2 x^{T} P \dot{x}-\zeta_{k}\left[S \zeta_{k}+2 Q x\left(t_{k}\right)\right] \\
& +2\left(T_{k}-\tau\right) \frac{d}{d t} \zeta_{k}\left[S \zeta_{k}+Q x\left(t_{k}\right)\right] \\
& +\left(T_{k}-2 \tau\right) x\left(t_{k}\right)^{T} U x\left(t_{k}\right) \\
& +\left(T_{k}-\tau\right) \Gamma_{1}+\Gamma_{2}
\end{aligned}
$$

where

$$
\begin{aligned}
\Gamma_{1}= & \sum_{i=0}^{N-1} \frac{d}{d t}\left\{t_{k}^{i}(t)\right\} \dot{x}\left(t_{k}^{i}(t)\right)^{T} R_{i} \dot{x}\left(t_{k}^{i}(t)\right) \\
& -\sum_{i=0}^{N-1} \frac{d}{d t}\left\{t_{k}^{i-1}(t)\right\} \dot{x}\left(t_{k}^{i-1}(t)\right)^{T} R_{i} \dot{x}\left(t_{k}^{i-1}(t)\right) \\
= & \sum_{i=0}^{N-1} \frac{N-i}{N} \dot{x}\left(t_{k}^{i}(t)\right)^{T} R_{i} \dot{x}\left(t_{k}^{i}(t)\right) \\
& -\sum_{i=0}^{N-1} \frac{N-i+1}{N} \dot{x}\left(t_{k}^{i-1}(t)\right)^{T} R_{i} \dot{x}\left(t_{k}^{i-1}(t)\right) \\
\Gamma_{2}= & -\sum_{i=0}^{N-1} \int_{t_{k}^{i-1}(t)}^{t_{k}^{i}(t)} \dot{x}(s)^{T} R_{i} \dot{x}(s) d s .
\end{aligned}
$$

Using the compact notations

$$
\begin{aligned}
\dot{x}(t) & =M_{0} X(t), & x(t) & =N_{0} X(t) \\
x\left(t_{k}\right) & =N_{2} X(t), & \dot{X}(t) & =M X(t) \\
\zeta_{k}(t) & =\Lambda_{12} X(t), & \frac{d}{d t} \zeta_{k}(t) & =\Lambda_{12} \frac{d}{d t} X(t)=\Lambda_{12} \Delta_{N}^{2} M X(t)
\end{aligned}
$$

with matrices defined in (13) we obtain

$$
\begin{aligned}
\dot{\mathcal{W}}\left(\tau, \varkappa_{k}\right) & \leq X^{T}\left\{2 N_{0}^{T} P M_{0}-\Lambda_{12}^{T}\left[S \Lambda_{12}+2 Q N_{2}\right]\right. \\
& \left.+\left(T_{k}-\tau\right)\left(\Lambda_{12} \Delta_{N}^{2} M\right)^{T}\left[2 S \Lambda_{12}+2 Q N_{2}\right]\right\} X \\
& +\left(T_{k}-\tau\right) \Gamma_{1}+\Gamma_{2} .
\end{aligned}
$$

Using (22), the term $\Gamma_{1}$ is rewritten as

$$
\begin{aligned}
\Gamma_{1} & =\dot{X}^{T} \operatorname{diag}\left\{R_{0}, \frac{N-1}{N} R_{1,0}, \ldots, \frac{1}{N} R_{N-1, N-2}, 0_{n}\right\} \dot{X} \\
& =\dot{X}^{T} \Delta_{N}^{T} \operatorname{diag}\left\{R_{0}, R_{1,0}, \ldots, R_{N-1, N-2}, R_{N-1}\right\} \Delta_{N} \dot{X}
\end{aligned}
$$

where $R_{i, i-1}=R_{i}-R_{i-1}, i=1, \ldots, N-1$. Note that, in the second expression, the matrix $R_{N-1}$ on the last diagonal block have been artificially introduced. This does not change the overall expression since the last diagonal block of $\Delta_{N}$ is zero. Finally, we obtain

$$
\begin{aligned}
\Gamma_{1} & =\dot{X}^{T} \Delta_{N}^{T}\left(\left[\begin{array}{cc}
\bar{R} & 0_{n N, n} \\
0_{n, n N} & 0_{n}
\end{array}\right]-\left[\begin{array}{cc}
0_{n} & 0_{n, n N} \\
0_{n N, n} & \bar{R}
\end{array}\right]\right) \Delta_{N} \dot{X} \\
& =\dot{X}^{T} \Delta_{N}^{T}\left[\Lambda_{1}^{T} \bar{R} \Lambda_{1}-\Lambda_{2}^{T} \bar{R} \Lambda_{2}\right] \Delta_{N} \dot{X} \\
& =\dot{X}^{T} \tilde{R} \dot{X}
\end{aligned}
$$

where the matrices $\Lambda_{1}, \Lambda_{2}$ and $\tilde{R}$ are defined in (13). 
Consider now the integral term $\Gamma_{2}$. Each integral term of the sum can be bounded as

$$
\begin{aligned}
-\int_{t_{k}^{i-1}}^{t_{k}^{i}} \dot{x}^{T}(s) R_{i} \dot{x}(s) d s \leq & -2 X^{T} Y_{i}\left[x\left(t_{k}^{i}(t)\right)-x\left(t_{k}^{i-1}(t)\right)\right] \\
& +\delta_{k}^{i}(t) X^{T} Y_{i} R_{i}^{-1} Y_{i}^{T} X
\end{aligned}
$$

where $Y_{i} \in \mathbb{R}^{n(N+1) \times n}, i=0, \ldots N-1$ are arbitrary matrices and $\delta_{k}^{i}(t):=t_{k}^{i}(t)-t_{k}^{i-1}(t)$. From the definition of the $t_{k}^{i}$ 's, it is easy to see that the quantity $\delta_{k}^{i}(t)$ is equal to $\left(t-t_{k}\right) / N=\tau / N$. Then summing the above inequalities over $i \in\{0, \ldots N-1\}$ leads to

$$
\begin{aligned}
\Gamma_{2} \leq & -2 X^{T}\left(\sum_{i=0}^{N-1} Y_{i}\left[x\left(t_{k}^{i}(t)\right)-x\left(t_{k}^{i-1}(t)\right)\right]\right) \\
& +\frac{\tau}{N} X^{T}\left(\sum_{i=0}^{N-1} Y_{i} R_{i}^{-1} Y_{i}^{T}\right) X .
\end{aligned}
$$

By introducing the matrix $Y=\left[Y_{0}, Y_{1}, \ldots, Y_{N-1}\right]$, the previous inequality are rewritten as

$$
\Gamma_{2} \leq-2 X^{T} Y \zeta_{k}+\frac{\tau}{N} X^{T} Y \bar{R}^{-1} Y^{T} X
$$

where $\bar{R}=\operatorname{diag}_{i=0, \ldots, N-1}\left\{R_{i}\right\}$.

Finally using (22), (23), (24) and (27) we obtain

$$
\begin{aligned}
\dot{\mathcal{W}}\left(\tau, \varkappa_{k}\right) \leq & X^{T}\left[2 N_{0}^{T} P M_{0}-\Lambda_{12}^{T}\left[S \Lambda_{12}+2 Q N_{2}\right]\right. \\
& -2 Y \Lambda_{12}+\left(T_{k}-\tau\right) M^{T} \tilde{R} M \\
& +\left(T_{k}-\tau\right)\left(\Lambda_{12} \Delta_{N}^{2} M\right)^{T}\left[2 S \Lambda_{12}+2 Q N_{2}\right] \\
& \left.+\left(T_{k}-2 \tau\right) N_{2}^{T} U N_{2}+\frac{\tau}{N} Y \bar{R}^{-1} Y^{T}\right] X
\end{aligned}
$$

or equivalently

$$
\begin{gathered}
\dot{\mathcal{W}}\left(\tau(t), \varkappa_{k}\right) \leq X(t)^{T} \Psi(\tau(t)) X(t) \\
\text { over } t \in\left[t_{k}, t_{k+1}\right) \text { with } \\
\Psi(\tau)=\Psi_{1}+\left(T_{k}-\tau\right) \Psi_{2}+\left(T_{k}-2 \tau\right) \Psi_{3}+\tau \Psi_{4}
\end{gathered}
$$

where the $\Psi_{i}$ 's for $i=1, \ldots, 3$ are given in (12) and $\Psi_{4}=Y^{T} \bar{R}^{-1} Y / N$. Then asymptotic stability is ensured if $\dot{\mathcal{W}}\left(\tau(t), \varkappa_{k}\right)<0$ over $t \in\left[t_{k}, t_{k+1}\right)$ or equivalently if

$$
\Psi_{1}+\left(T_{k}-\tau\right) \Psi_{2}+\left(T_{k}-2 \tau\right) \Psi_{3}+\tau \Psi_{4} \prec 0
$$

for all $\left(\tau, T_{k}\right) \in\left[0, T_{k}\right] \times\left[T^{-}, T^{+}\right]$. We have thus obtained a semi-infinite matrix inequality depending affinely on both $\tau$ and $T_{k}$.

Part 4. Turning the above semi-infinite dimensional problem into an equivalent finite dimensional one is easy in this case. Indeed, since $\Psi(\tau)$ depends linearly on $\tau$ then, using a convexity argument, it is necessary and sufficient to check the feasibility of the condition on the vertices of the set $\left[0, T_{k}\right]$, that is, on the set $\left\{0, t_{k}\right\}$. This yields the underlying matrix inequality problem

$$
\Psi_{1}+T_{k}\left(\Psi_{3}+\Psi_{4}\right) \prec 0, \quad \Psi_{1}+T_{k}\left(\Psi_{2}-\Psi_{4}\right) \prec 0
$$

which is also linear in $T_{k}$. Since the sampling period is not constant and belongs to $\left[T^{-}, T^{+}\right]$, using the same reasoning as above, we need to check the feasibility of the above at the vertices of the set $\left[T^{-}, T^{+}\right]$, i.e. for values in $\left\{T^{-}, T^{+}\right\}$. This leads to the conditions of Theorem 2 after some Schur complements (to linearize the rational term $\left.\Psi_{4}\right)$. The proof is complete.

The following immediate corollary deals with constant and known sampling period.

Corollary 3. The system (1)-(2) is asymptotically stable with constant sampling period $T$ if there exist constant

\begin{tabular}{|c|l|l|}
\hline Theorems & Ex.1 & Ex.2 \\
\hline \hline$[$ Fridman et al., 2004] & {$[0,0.869]$} & {$[0,0.99]$} \\
{$[$ Naghshtabrizi et al., 2008 $]$} & {$[0,1.113]$} & {$[0,1.99]$} \\
{$[$ Fridman, 2010] } & {$[0,1.695]$} & {$[0,2.03]$} \\
{$[$ Liu and Fridman, 2009] } & {$[0,1.695]$} & {$[0,2.53]$} \\
[Seuret, 2010] & {$[0,1.721]$} & {$[0,2.51]$} \\
\hline \hline Th.2, $N=1$ & {$[0,1.721]$} & {$[0,2.51]$} \\
\hline Th.2, $N=3$ & {$[0,1.727]$} & {$[0,2.62]$} \\
\hline Th.2, $N=5$ & {$[0,1.728]$} & {$[0,2.64]$} \\
\hline Table 1. Interval of allowable asynchronous
\end{tabular}
samplings for Examples 1 and 2.

matrices $P=P^{T} \succ 0, R_{i}=R_{i}^{T} \succ 0, i=0, \ldots, N-1$ and $U=U^{T} \in \mathbb{R}^{n \times n}, S=S^{T} \in \mathbb{R}^{n N \times n N}, Q \in \mathbb{R}^{n N \times n}$ and $Y \in \mathbb{R}^{n(N+1) \times n N}$ such that the LMIs

$$
\begin{array}{cc}
\Psi_{1}+T \Psi_{2}+T \Psi_{3} & \prec 0 \\
{\left[\begin{array}{cc}
\Psi_{1}-T \Psi_{3} & T Y \\
\star & -\alpha \bar{R}
\end{array}\right]} & \prec 0
\end{array}
$$

hold where the matrices $\Psi_{1}, \Psi_{2}$ and $\Psi_{3}$ are given in Theorem 2 and $\alpha=N T$.

Remark 4.1. Note that taking $N=1$, the functionals defined in [Seuret, 2010] and in [Fridman, 2010] (but with $U=0)$ are retrieved.

It is also important to mention that the problem of pathological sampling is automatically included in the conditions. Indeed, the set $\left[T^{-}, T^{+}\right]$cannot contain such sampling period since in this case, the matrix $\tilde{A}\left(T^{o}\right)$, for some pathological sampling period $T^{o}$, is not Schur and hence conditions of Theorem 2 and Corollary 3 cannot be satisfied. Thus there is no need of considering pathological sampling periods separately.

\section{EXAMPLES}

To illustrate the approach let us consider system (1)-(2) with matrices

(1) Ex.1 [Fridman et al., 2004, Naghshtabrizi et al., 2008]

$$
A=\left[\begin{array}{cc}
0 & 1 \\
0 & -0.1
\end{array}\right], \quad B K=\left[\begin{array}{cc}
0 & 0 \\
-0.375 & -1.15
\end{array}\right]
$$

(2) Ex.2 [Fridman, 2010]

$$
A=\left[\begin{array}{cc}
-2 & 0 \\
0 & -0.9
\end{array}\right], \quad B K=\left[\begin{array}{cc}
-1 & 0 \\
-1 & -1
\end{array}\right]
$$

(3) Ex.3 [Gu et al., 2003, Michiels et al., 2004]

$$
A=\left[\begin{array}{cc}
0 & 1 \\
-2 & 0.1
\end{array}\right], \quad B K=\left[\begin{array}{ll}
0 & 0 \\
1 & 0
\end{array}\right]
$$

Tables 1 and 2 summarize the results obtained in the literature and using Theorem 2. A first remark concerns the reduction of conservatism with respect to the existing results dealing with the continuous-time approaches when $N=1$ (except for example 2 with the method of [Liu and Fridman, 2009]). We can also see that, as noticed in Remark 4.1, Theorem 2 with $N=1$ leads to identical results as those of [Seuret, 2010]. Moreover, as expected when the fragmentation order $N$ is increased, the conservatism is reduced.

The system of Example 3 exhibits a singular behavior. Indeed, while the corresponding continuous-time system $\dot{x}(t)=(A+B K) x(t)$ is unstable, and so is discrete-time for sufficiently small sampling period, it becomes stable when 


\begin{tabular}{|c|c|}
\hline Theorems & Ex.3 \\
\hline \hline [Fridman et al., 2004] & - \\
[Naghshtabrizi et al., 2008] & - \\
[Fridman, 2010] & - \\
[Liu and Fridman, 2009] & - \\
{$[$ Seuret, 2010] } & {$[0.40,1.11]$} \\
\hline Th.2, $N=1$ & {$[0.40,1.11]$} \\
\hline Th. $2, N=3$ & {$[0.40,1.28]$} \\
\hline Th. $2, N=5$ & {$[0.40,1.31]$} \\
\hline
\end{tabular}

Table 2. Interval of allowable asynchronous samplings for Example 3.

the sampling period is larger than a threshold, determined to be 0.4 . This type of systems cannot be considered with previous techniques, see e.g [Naghshtabrizi et al., 2008, Liu and Fridman, 2009, Fridman, 2010] since the stability of the corresponding continuous-time is a necessary condition (encoded in the LMIs). It is indeed well-known that if the system is stable in continuous-time, then it will be stable for a sufficiently small sampling period, the converse is, in general, not true, as nicely illustrated by this example.

The provided method allows to consider these systems due to the presence of the indefinite matrix $U$ in LMIs (11) and (33) which are obtained when $\dot{\mathcal{W}}\left(\tau, \varkappa_{k}\right)$ is evaluated at $\tau=0$. Indeed, when $\tau=0$, the system is pointwisely a continuous-time system and becomes a sampled-data system when $\tau>0$. The matrix $U$ allows to 'compensate' the instability of the system in the region $[0,0.4]$. Note that in this region the Lyapunov function $V$ increases to finally decrease in the region $[0.4,1.31]$ to make the Lyapunov function globally decreasing (or decreasing in mean) over $[0, \theta]$ with $\theta \in(0.4,1.31]$.

\section{ACKNOWLEDGMENTS}

The authors would like to thank the anonymous reviewers who helped to improve this work.

\section{REFERENCES}

C. Briat. Control and Observation of LPV Time-Delay Systems. PhD thesis, Grenoble-INP, 2008. URL http://www. briat.info/thesis/PhDThesis.pdf.

T. Chen and B. Francis. Optimal Sampled-Data Control Systems. Berlin: Springer, 1995.

T. Chen and B.A. Francis. On the $L^{2}$ induced norm of a sampled-data system. Systems \& Control Letters, 15: 211-219, 1990.

E. Fridman. A refined input delay approach to sampleddata control. Automatica, 46(2):421-427, 2010.

E. Fridman, A. Seuret, and J. P. Richard. Robust sampleddata stabilization of linear systems: An input delay approach. Automatica, 40:1441-1446, 2004.

H. Fujioka. A discrete-time approach to stability analysis of systems with aperiodic sample-and-hold devices. IEEE Transactions on Automatic Control, 54(10):24402445, 2009a.

H. Fujioka. Stability analysis of systems with aperiodic sample-and-hold devices. Automatica, 45:771-775, 2009b.

H. Fujioka, T.akai, and L. Hetel. A switched lyapunov functional approach to stability analysis of nonuniformly sampled-data systems. In American Control
Conference, Marriott Waterfront, Baltimore, MD, USA, 2010.

F. Gouaisbaut and D. Peaucelle. Delay dependent robust stability of time delay-systems. In $5^{\text {th }}$ IFAC Symposium on Robust Control Design, Toulouse, France, 2006.

F .Gouaisbaut and D. Peaucelle. Delay-dependent stability analysis of linear time delay systems. In 6th IFAC Workshop on Time Delay Systems, L'Aquila, Italy, 2006.

K. Gu, V.L. Kharitonov, and J. Chen. Stability of TimeDelay Systems. Birkhäuser, 2003.

J.P. Hespanha, P. Naghshtabrizi, and Y. Xu. A survey of recent results in networked control systems. Proceedings of the IEEE, 95(1):138-162, 2007.

L. Hetel, J. Daafouz, and C. Iung. Analysis and control of LTI and switched systems in digital loops via an eventbased modelling. International Journal of Control, 81: 1125-1138, 2008.

K. Liu and E. Fridman. Discontinuous Lyapunov functionals for linear systems with sawtooth delays. In Proc. of the $8^{\text {th }}$ IFAC Workshop on Time-Delay Systems, Sinaia, Romania, September 2009.

W. Michiels, S.-I. Niculescu, and L. Moreau. Using delays and time-varying gains to improve the static output feedback stabilizability of linear systems : a comparison. IMA Journal of Mathematical Control and Information, 21(4):393-418, 2004.

L. Mirkin. Some remarks on the use of time-varying delay to model sample-and-hold circuits. IEEE Transactions on Automatic Control, 52(6):1109-1112, 2007.

L.A. Montestruque and P.J. Antsaklis. Stability of modelbased networked control systems with time-varying transmission times. IEEE Transactions on Automatic Control, 49(9):1562-1572, 2004.

P. Naghshtabrizi, J.P. Hespanha, and A.R. Teel. Exponential stability of impulsive systems with application to uncertain sampled-data systems. Systems \& Control Letters, 57:378-385, 2008.

Y. Oishi and H. Fujioka. Stability and stabilization of aperdiodic sampled-data control systems: an approach using robust linear matrix inequalities. In 48th Conference on Decision and Control, pages 8142-8147, Shanghai, China, 2009.

M.M. Peet, A. Papachristodoulou, and S. Lall. Positive forms and stability of linear time-delay systems. SIAM Journal on Control and Optimization, 47(6):3227-3258, 2009.

A. Seuret. Stability analysis for sampled-data systems with a time-varying period. In 48th Conference on Decision and Control, pages 8130-8135, Shanghai, China, 2009.

A. Seuret. A novel stability analysis of linear systems under asynchronous samplings. Accepted to Automatica, 2010.

Y.S. Suh. Stability and stabilization of nonuniform sampling systems. Automatica, 44:3222-3226, 2008.

V. Suplin, E. Fridman, and U. Shaked. Sampled-data $\mathcal{H}_{\infty}$ control and filtering: Non uniform uncertain sampling. Automatica, 43:1072-1083, 2007.

W. Zhang, M.S. Branicky, and S.M. Phillips. Stability of networked control systems. IEEE Control Systems Magazine, 21(1):84-99, 2001. 